\title{
Lamin A/C mutations associated with familial and sporadic cases of dilated cardiomyopathy in Koreans
}

\author{
Kyuyoung Song ${ }^{1,5}$, Marie-Pierre Dubé ${ }^{4}$, \\ Jiyoung Lim ${ }^{1}$, Ilsun Hwang ${ }^{2}$, \\ Inchul $\mathrm{Lee}^{2}$ and Jae-Joong $\mathrm{Kim}^{3,5}$ \\ ${ }^{1}$ Department of Biochemistry and Molecular Biology \\ ${ }^{2}$ Department of Pathology \\ ${ }^{3}$ Department of Internal Medicine \\ Asan Medical Center \\ University of Ulsan College of Medicine \\ Seoul 138-736, Korea \\ ${ }^{4}$ Institut de Cardiologie de Montréal \\ Université de Montréal \\ Quebec, Canada \\ ${ }^{5}$ Corresponding authors: Tel, 82-2-3010-3154; Fax, 82-2-3010-5918; \\ E-mail, jjkim@amc.seoul.kr (for J.J.K.) and \\ Tel, 82-2-3010-4277; Fax, 82-2-3010-4248; \\ E-mail, kysong@amc.seoul.kr (for K.Y.S.)
}

Accepted 4 January 2007

Abbreviations: ACE, angiotensin I-converting enzyme; DCM, dilated cardiomyopathy; FDC, familial dilated cardiomyopathy; LMNA, lamin $A / C$; LVEDD, left ventricular end-diastolic dimension

\begin{abstract}
Dilated cardiomyopathy (DCM) is characterized by cardiac dilation and systolic dysfunction. So far sixteen genes have been shown to cause autosomal dominant familial dilated cardiomyopathy (FDC). We identified a large Korean family from the Jeju island showing a clear Mendelian inheritance of FDC. A genomewide linkage scan at $9 \mathrm{cM}$ marker density identified a peak multipoint LOD score of 2.82 at D1S195. Haplotyping of the region with 15 additional markers defined a candidate interval that included a known candidate gene encoding the lamin $A / C$ (LMNA). Sequencing of the LMNA exons revealed one missense mutation at C568T (Arg190Trp) in the $\alpha$-helical rod domain of the LMNA gene cosegregating with FDC with conduction-system disease. The same mutation was found in patients of another Korean family with FDC without conduction-system disease. Upon screening 14 sporadic DCM cases, we found three LMNA mutations including a case having a previously described (Glu161Lys) mutation and two having novel mutations (Glu53Val and
\end{abstract}

Glu186Lys). Our results suggest that variable genotypes of laminopathy are implicated in not only familial but also considerable proportion of sporadic DCM.

Keywords: Asian continental ancestry group; cardiomyopathy, dilated; genetics; heart conduction system; lamins; mutation

\section{Introduction}

Dilated cardiomyopathy (DCM) characterized by cardiac dilation and systolic dysfunction represents a heterogeneous group of inherited and acquired disorders (for review, see Ahmad et al., 2005; Burkett and Hershberger, 2005). About $35 \%$ of individuals with DCM have a familial form of the disease (FDC) (Grunig et al., 1998). FDC is highly heterogeneous disease genetically. Mutations in 25 chromosome loci have been described for DCM with or without additional clinical manifestations (for review, see Burkett and Hershberger, 2005). Among the $16 \mathrm{mu}-$ tated genes associated with DCM, the human nucleoplasmic lamin A/C (LMNA) gene has been reported in forms associated with conduction-system disease with or without skeletal muscle myopathy.

In the present study we performed a linkage analysis followed by a candidate gene screening to localize the disease gene in a Korean family affected with an autosomal dominant severe DCM with conduction-system disease. By a mutation screen of the coding sequence of the LMNA gene on all family members, we identified a previously described missense mutation (Arg190Trp) in the rod domain of the LMNA gene that cosegregated with the disease in the family. We also found the same mutation in patients of another Korean family with FDC without conduction-system disease. Previously ten mutations (R60G, L85R, K97E, E111X, E161K, Arg190Trp, N195K, E203G, E317K, R377H) in the rod domain of the LMNA gene were reported to be associated with DCM through disorganization of the lamina (Fatkin et al., 1999; Arbustini et al., 2002; Sebillon et al., 2003). Upon screening additional 14 patients, we found a previously described (Glu161Lys) mutation and two novel (Glu53Val and Glu186Lys) mutations. Our results show that LMNA mutations are associated with FDC and DCM in Koreans. 


\section{Materials and Methods}

\section{Clinical evaluations}

We studied 35 subjects through 2 generations from one Korean family (family A), 2 subjects in another family (family B), and 14 sporadic cases without familial background. Six members of family $A$, the 2 tested patients in family $B$, and 14 sporadic cases showed phenotypically manifested DCM. The diagnosis of DCM was made based on previously accepted criteria (Mestroni et al., 1999). Clinical evaluations included a detailed history, physical examination, 12-lead electrocardiogram, chest x-ray and echocardiogram.

\section{Genotyping}

Genomic DNA was isolated from peripheral blood samples following standard procedures (Lee et al., 2001). Signed informed consent was obtained from all study subjects or their tutors according to the institutional review board policies. The genomewide scan was performed by using 392 highly polymorphic microsatellite markers spaced at an average distance of $9.0 \mathrm{cM}$ at the McGill University and Genome Quebec Innovation Centre. Each marker was amplified individually followed by pooling of up to 8 markers together in panels. The genotyping results were obtained using an $\mathrm{ABI}-3700$ or 3730
DNA Analyzer (Applied Biosystems). This study was approved by the Ethical Committee of the Asan Medical Center.

\section{Linkage analysis}

Two point linkage analyses were carried-out by using the MLINK routine of FASTLINK v4.1P. Multipoint linkage and haplotyping were done by using SIMWALK2 version 2.90 on Linux. For the genomewide scan, we used DNA samples from 25 pedigree members, of which six were from affected members. The pedigree provided sufficient power to detect linkage in a $10 \mathrm{cM}$ scan with an estimated average linked LOD score of 1.12. The inheritance model was defined as dominant with 0.95 penetrance for pedigree members above age 45, 0.001 phenocopy rate, and a disease allele frequency of 0.001 . All individuals younger than 45 years of age (except the spouses) were coded as phenotype unknown (Figure 1, family A) for the purpose of the linkage analyses. Marker allele frequencies were uniformly distributed to observed alleles counts due to the lack of reference samples for frequency estimation. Considering that the pedigree contains a high proportion of affected members, this should have a conservative effect on the LOD score statistics. Chromosome $X$ was not considered for linkage due to the high number of male to male transmissions in the pedigree.

A

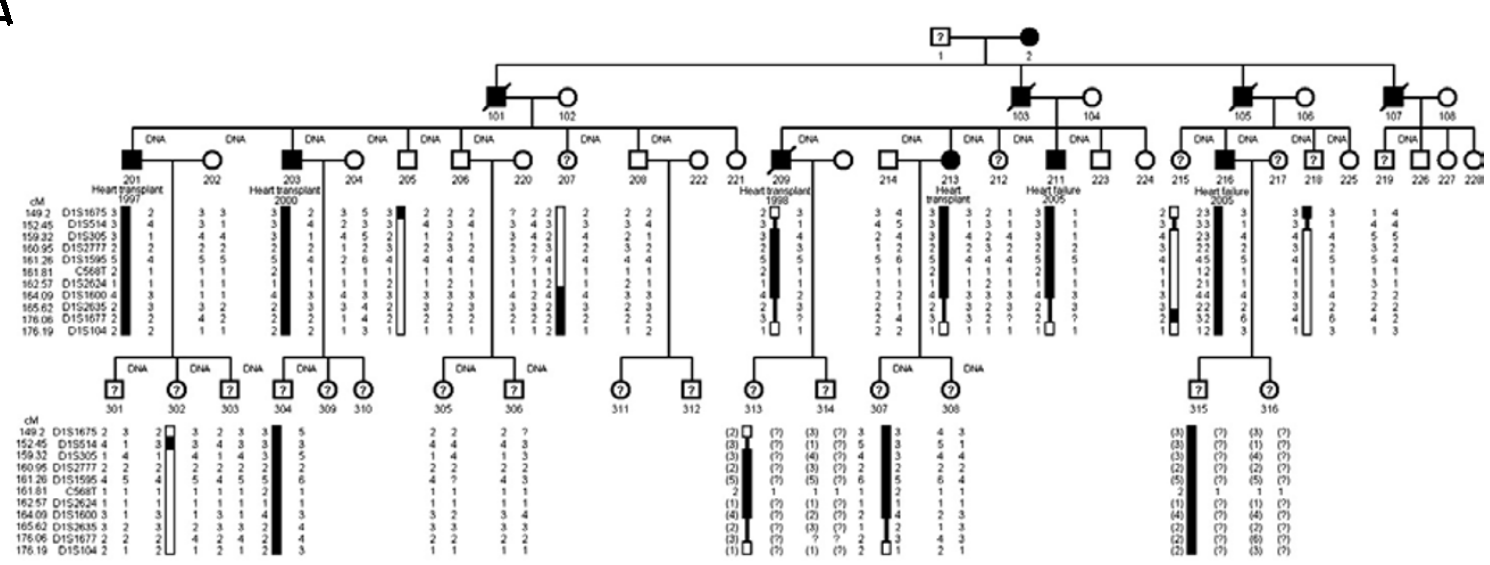

B

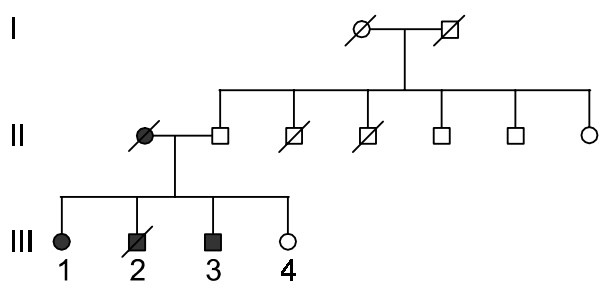

Figure 1. Pedigree of the families. Affected status is indicated by filled symbols, unaffected by clear symbols. Diagonal lines indicate deceased individuals. (A) Pedigree structure and haplotypes for family A. (B) Pedigree structure for family B. 


\section{Identification of mutations in the LMNA gene}

All 12 exons and exon-intron junctions of the LMNA gene were amplified by PCR in the DNA sample from individual 201 of family $A$ and were analyzed by direct sequencing using a MegaBACE 1000 sequencer (Amersham Biosciences, Backinghamshire, England) following the manufacturer's instructions (primer sequences available upon request). One variant was identified in individual 201 from family $A$ by sequence analysis of both strands. The presence or absence of the sequence variant was ascertained in all 35 pedigree members from the family $A, 2$ siblings with FDC from family $B, 14$ additional sporadic cases, and 250 unrelated Korean controls by sequence analysis.

\section{Results}

\section{Linkage analyses}

The family with autosomal dominant inheritance of

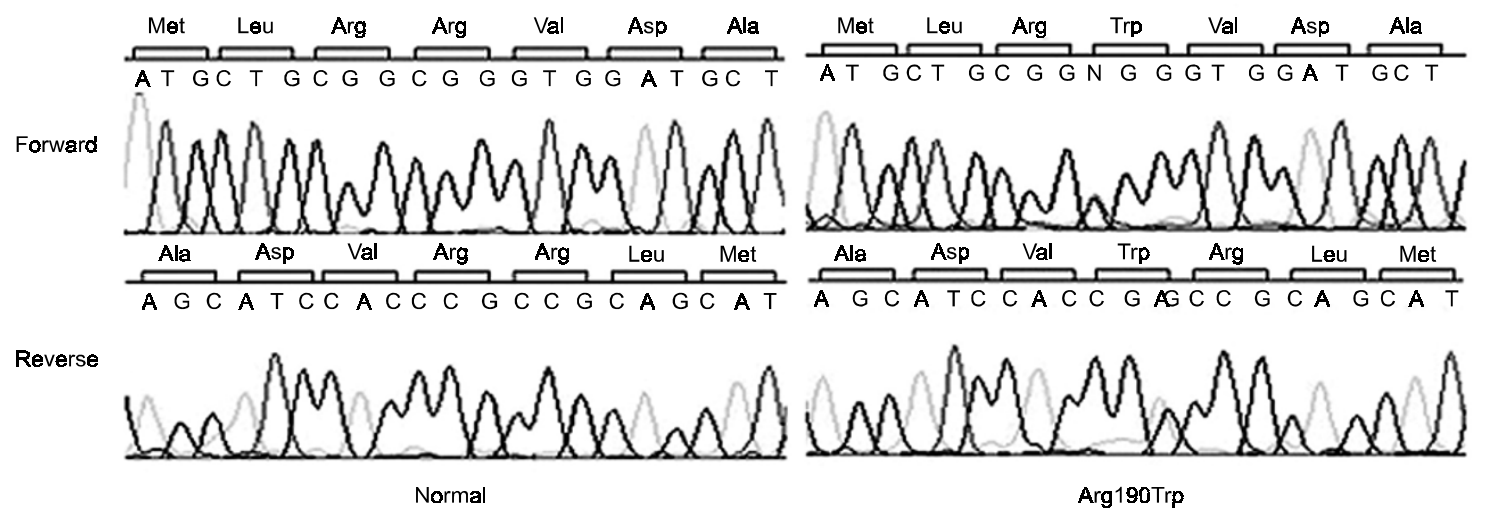

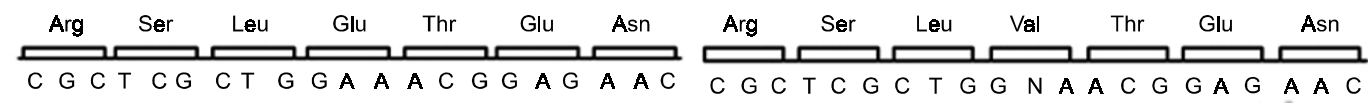
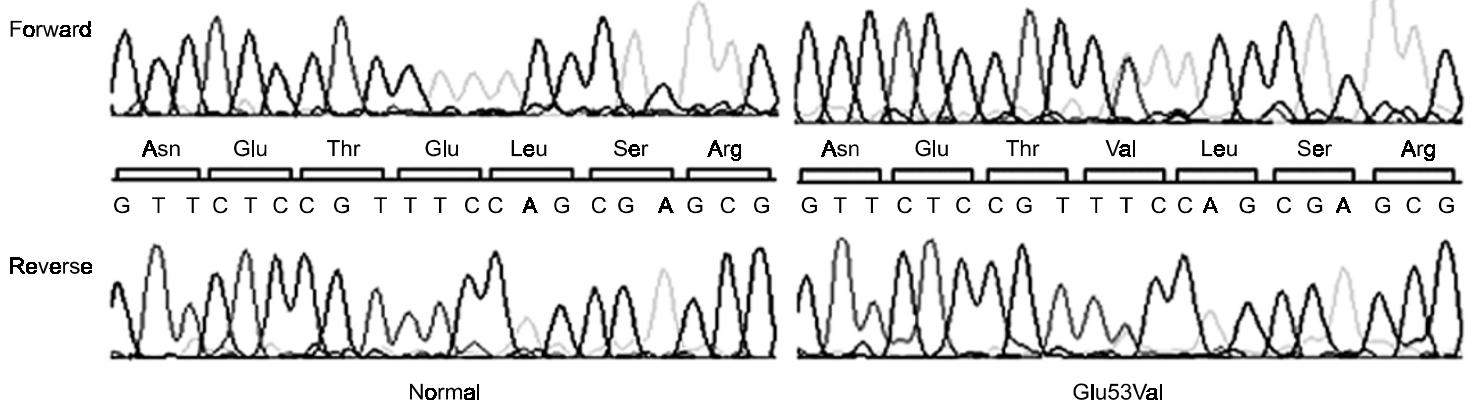

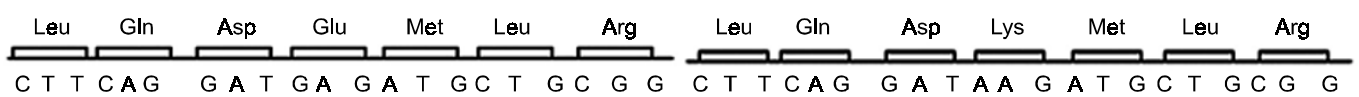
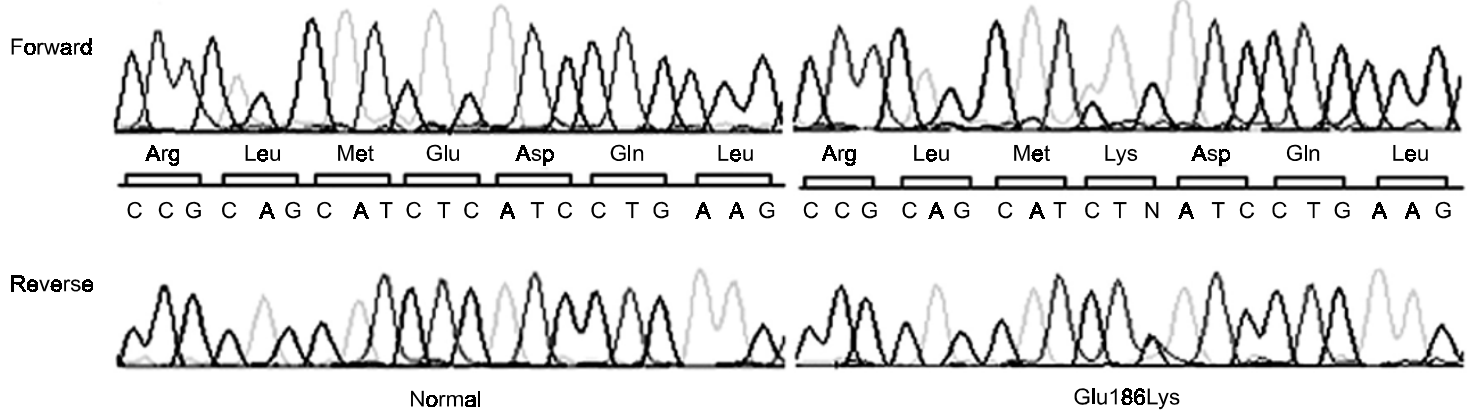

Figure 2. The direct sequencing results (both forward and reverse sequence) indicate the corresponding nucleotide substitutions. 
DCM consisted of 4 generations including 2 living generations totaling 50 subjects and 29 subjects were fully genotyped (Figure 1A). There were no clinical data available from the deceased individuals of the 2 nd generation of which all five males died in the forth decades of life. Affected relatives were identified in 3 of 6 branches of the pedigree. Of 24 first-degree relatives, 5 males and one female were affected with idiopathic dilated cardiomyopathy. A genomewide screen was initiated, and a total of 392 micorsatellite markers were genotyped. Five regions with LOD scores greater than 1 were found in two-point linkage on chromosomes 1, 2, 7, 13 and 21. Linkage to D1S1595 on chromosome 1 was strongly supported by the multipoint linkage with a $L O D=2.82$, the highest finding in the study. Haplotyping over the chromosome 1 region revealed a candidate interval that included the known candidate gene, $L M N A$, encoding LMNA. To help resolve the difficulty in phase construction due to the unavailability of generations I and II and to define the recombinant boundaries for the candidate gene region, fifteen additional markers were added in the D1S1675 to D1S2107 interval. This allowed a $27 \mathrm{cM}$ candidate interval $(45.9 \mathrm{Mb})$ including the centromere and flanked by marker D1S1675 and D1S104.

\section{Mutation analysis of a candidate gene}

The LMNA gene was considered candidate for mutation in this family because the most frequent DCM-associated mutations have been reported in the LMNA gene located in chromosome 1q21.2. Twelve exon sequences of the LMNA gene were analyzed in proband, individual 201, of family A and a unique sequence variant which cause amino acid change at position 190 from Arg to Trp in exon 3 was identified (Figure 2). This mutation, Arg190Trp, was previously reported (Arbustini et al., 2002; Anan et al., 2002; Hermida-Prieto et al., 2004) and predicted to alter residues in the $\alpha$-helical rod domain of the peptide. Previously 10 missense mutations identified in the $\alpha$-helical rod domain of the LMNA were reported to be disease-causing mutations in Caucasians with FDC and DCM. Then we also performed a mutation screen of the coding sequence of the LMNA gene on sibling samples with FDC from the other family and identified the same mutation, Arg190Trp (Figure 1B). By screening of additional 14 sporadic cases which showed nuclear detachment with evident cytoplasmic degeneration, we found three cases with LMNA mutations including a previously described (Glu161Lys) mutation and two novel mutations, Glu53Val and Glu186Lys (Figure 2 ). The fact that these variants were present in all the probands of the families and sporadic cases but not in 504 chromosomes from normal individuals demonstrates that these variants are not a common polymorphism.

\section{Clinical features of the LMNA mutation}

There were 8 ( 6 from family $A$ and 2 from family $B$ ) phenotypically manifested FDC patients from 2 families. The pedigrees are shown in Figure 1. The age at disease manifestation was early in middle age (mean 39 years; range 36-43) (Table 1). Five of 8 underwent heart transplantation $(201,203,209$, 213 of family A, III-3 of family B) because of advanced heart failure. They do well currently except individual 209 of famlily A who died. Mean time from the detection of heart failure to transplantation was 2.4 yrs (range 1.5-4 yrs). Three other patients (211, 216 of family A and III-1 of family B) are receiving ACE inhibitor and beta-blocker therapy for heart failure. Their initial ejection fraction and left ventricular end-diastolic dimension (LVEDD) were 24\%, $6.1 \mathrm{~cm}, 34 \%, 6.6 \mathrm{~cm}$, and $27 \%, 5.9 \mathrm{~cm}$ respectively.

In family A, 5 patients showed conduction-system disease as previously described (Fatkin et al., 1999). They showed sinus-node dysfunction and/or disturbances in atrioventricular conduction (sinus bradycardia, bundle branch block and/or first-degree block). Atrial fibrillation/flutter which was paroxysmal atrial flutter was present in only one patient (213, family A). None of two patients in family $B$ had any

Table 1. Clinical characteristics of 11 manifested dilated cardiomyopathy patients.

\begin{tabular}{|c|c|c|c|c|}
\hline Patient ID & Sex/Age & Course & ECG & Af \\
\hline \multicolumn{5}{|l|}{ Family A } \\
\hline 201 & $\mathrm{M} / 43^{*}$ & Heat TPL & $\mathrm{C}-\mathrm{RBBB}, 1^{\circ} \mathrm{AVB}$ & No \\
\hline 203 & $\mathrm{M} / 43^{*}$ & Heat TPL & $\begin{array}{l}1^{\circ} \mathrm{AVB}, \\
\text { Sinus bradycardia }\end{array}$ & No \\
\hline 209 & $M / 38^{*}$ & Heat TPL & C-RBBB & No \\
\hline 213 & $\mathrm{~F} / 40^{*}$ & Heat TPL & $\mathrm{C}-\mathrm{RBBB}, 1^{\circ} \mathrm{AVB}$ & Yes \\
\hline 211 & $\mathrm{M} / 40^{*}$ & Medical Tx & Normal & No \\
\hline 216 & $\mathrm{M} / 37^{*}$ & Medical Tx & Sinus bradycardia & No \\
\hline \multicolumn{5}{|l|}{ Family B } \\
\hline III-1 & $\mathrm{F} / 37^{*}$ & Medical Tx & Normal & No \\
\hline III-3 & $M / 36^{*}$ & Heat TPL & Normal & No \\
\hline \multicolumn{5}{|c|}{ Sporadic cases } \\
\hline Case 1 & $\mathrm{M} / 30$ & Heat TPL & C-LBBB & Yes \\
\hline Case 2 & $\mathrm{M} / 42$ & Heat TPL & $\begin{array}{l}1^{\circ} \mathrm{AVB}, \\
\text { Sinus bradycardia }\end{array}$ & No \\
\hline Case 3 & $\mathrm{M} / 42$ & Heat TPL & High grade AVB & Yes \\
\hline
\end{tabular}

$\mathrm{Age}^{*}$, age at clinical presentation (years old); TPL, transplantation; C-RBBB, complete right bundle branch block; C-LBBB, complete left bundle branch block; $1^{\circ} \mathrm{AVB}$, the first degree Atrioventricular block; Af, atrial fibrillation; Medical Tx, medical treatment for heart failure 
conduction-system disease.

There are 4 other family members in the 4 th generation who have the same gene mutation but without phenotypically manifested familial dilated cardiomyopathy yet $(304,313,307,315$ in Figure $1 \mathrm{~A})$. They do not show any conduction-system abnormalities. Their ages were 8, 16, 21 and 26 years.

None of family members including 8 phenotypically manifested DCM patients showed any evidence of skeletal muscle involvement such as skeletal muscle dysfunction, no weakness or wasting of skeletal muscle and no joint contracture. The serum creatine kinase level of all patients was in normal range.

All 3 patients of sporadic cases with LMNA mutation showed conduction-system disease (Table 1). In one patient with novel mutation (Glu53Val) was treated with permanent cardiac pacemaker due to high grade atrioventricular block at the age of 47 (case 3 ). The ages of onset were 30,42 and 42 years. None showed skeletal muscle abnormalities. Two patients showed so rapid progression of heart failure that they underwent heart transplantation within 2 years. But in case 3 , the progression was slow and he got heart transplantation after 8 years. In three families, no one else had heart failure.

\section{Discussion}

The pattern of inheritance of FDC includes autosomal dominant, autosomal recessive, X-linked, and mitochondrial inheritance. Autosomal dominant form of FDC is the most frequent and can be grouped into either a pure DCM phenotype or DCM with conduction system disease. So far 25 genomic loci have been mapped in different DCM families, and 20 individual disease genes have been identified and screened for mutations in affected individuals (for review, see Burkett et al., 2005). The genes involved in DCM encode a broad variety of proteins including sarcomeric, Z-disk-associated, sarcolemma cytoskeleton, intermediate filament, nuclear membrane, and calcium cycle proteins. Although numerous genes causing monogenic subtypes of DCM were identified, the extensive heterogeneity of disease phenotypes associated with mutations in a single gene or even in the same codon of that gene is not understood. It could be due to independent genetic factors or to environmental factors. Thus, we decided to carry out a genome-wide screen for an exclusion mapping.

The candidate region identified in genome-wide screen was flanked by markers D1S1675 and D1S104 in a $27 \mathrm{cM}$ interval. From the region, Lamin $\mathrm{A} / \mathrm{C}$, a nuclear intermediate filament, is a best candidate for FDC because it is well known that mutations in the LMNA gene cause a variety of human diseases including Emery-Dreifuss muscular dystrophy, dilated cardiomyopathy and HutchinsonGilford progeria syndrome (Bonne et al., 1999; 2003; Brodsky et al., 2000; Raharjo et al., 2001; Burke and Stewart, 2002). Furthermore, the most frequent DCMassociated mutations have been reported in the LMNA gene (Fatkin et al., 1999; Brodsky et al., 2000; Jakobs et al., 2001; Arbustini et al., 2002; Hershberger et al., 2002; Sebillon et al., 2003; Taylor et al., 2003; Karkkainen et al., 2004). DCM-associated mutations in the LMNA gene have been reported to cause very similar phenotypes characterized by atrial fibrillation, conduction system disease, ventricular dysrhythmia, need for pacemaker implantation, and high risk of sudden death. Recently, Taylor et al. (2003) showed that DCM patients with lamin $A / C$ mutations had a poor prognosis compared with other DCM patients. They also found that lamin A/C mutations were associated with supraventricular arrhythmias, conduction-system disease, mild DCM (slightly dilated left ventricle), and the presence of skeletal muscle disease.

Eleven mutations of Lamin A/C associated with cardiac abnormalities are located in the central rod domain of the protein (Fatkin et al., 1999; Arbustini et al., 2002; Sebillon et al., 2003). Of four mutations we found in the Korean families and sporadic cases, E53V and E186K were new missense mutations and the rest were not. The Arg190Trp mutation we found in the Korean families is previously described and reported to be located in the central rod domain (Anan et al., 2002; Arbustini et al., 2002; HermidaPrieto et al., 2004). This mutation has been associated with severe forms of familial DCM with conduction system disease but atrioventricular conduction defects were absent in the family with this mutation. Additional two mutations, E53V and E186K, we found in the two sporadic cases were also to be located in the central rod domain. Previously, it was shown that mutations of the LMNA gene frequently cause a particular phenotype DCM with conduction disease (Fatkin et al., 1999; Brodsky et al., 2000; Jakobs et al., 2001; Taylor et al., 2003; Karkkainen et al., 2004). Subsequently a mouse line expressing Lmna-N195K variant of the A-type lamins with an asparagine-to-lysine substitution at amino acid 195 showed characteristics consistent with DCM with conduction system disease (Mounkes et al., 2005).

In the Korean families we studied, the LMNA Arg190Trp mutation caused FDC in 2 families. This LMNA mutation in Korean families showed some different features. First, conduction-system disease was identified in 5 of 6 affected members in only one family. The same mutation did not cause conduction- 
system disease in the other family. One member who had heart transplantation did not show any conduction-system disease even at the time of heart transplantation. The other one member detected heart failure 3 years ago but has not shown any conduction-system disease yet. Both families had DCM with rapid progression in young men, and the age of onset was also similar within each family. Second, our patients had the LMNA Arg190Trp mutation but atrioventricular conduction disturbance was present in $3(38 \%)$ even though it was only first degree block and other conduction-system disease was present in the other 2 patients. Total 5 of 8 affected members $(63 \%)$ had conduction-system disease. This figure is higher than previously reported (Anan et al., 2002; Arbustini et al., 2002). Third, the incidence of atrial fibrillation or flutter was low $(12 \%$, only 1 episode of paroxysmal atrial flutter in 1 patient) in our study patients. This figure is lower than that of previously described (59\%) (Fatkin et al., 1999).

All 3 sporadic cases had conduction-system disease and 2 showed atrial fibrillation. Based on our data, it is not clear whether mutations in LMNA are also responsible for conduction-system disease. However, our results support the convenience of using lamin $\mathrm{A} / \mathrm{C}$ as a candidate gene in FDC with or without conduction abnormalities.

Since at least 30 to $40 \%$ of cases of DCM are inherited and FDC cannot be predicted on a clinical or morphological basis only, necessity of family screening is recognized by caregivers and scientists. However, it has also become clear that the disease is genetically and phenotypically highly heterogeneous. Multiple genes have been identified, most patients having private mutations, and the same mutation causing different phenotypes. The advances in the genetics of FDC will allow improved diagnosis, prevention and genetic counseling, and represent the basis for the development of new therapies.

\section{Acknowledgement}

This work was supported by the grants to J.-J. Kim (\#2003297) from the Asan Institute for Life Sciences, Seoul, Korea, and K. Song from the Korean HapMap Project of MOST (Ministry of Science and Technology of Korea). M.-P. Dubé is supported by the Fonds de Recherche en Santé du Québec (FRSQ).

\section{References}

Ahmad F, Seidman JG, Seidman CE. The genetic basis for cardiac remodeling. Annu Rev Genomics Hum Genet 2005;6: $185-216$
Anan R, Niimura H, Sasaki T, Seidman JG, Seidman CE, Minagoe S, Tei C. A novel lamin A/C missense mutation in a family with autosomal dominant dilated cardiomyopathy with conduction abnormalities. J Am Coll Cardiol 2002;39:s136

Arbustini E, Pilotto A, Repetto A, Grasso M, Negri A, Diegoli M, Campana C, Scelsi L, Baldini E, Gavazzi A, Tavazzi L. Autosomal dominant dilated cardiomyopathy with atrioventricular block: a lamin A/C defect-related disease. J Am Coll Cardiol 2002;39:981-90

Bonne G, Di Barletta MR, Varnous S, Becane HM, Hammouda EH, Merlini L, Muntoni F, Greenberg CR, Gary F, Urtizberea JA, Duboc D, Fardeau M, Toniolo D, Schwartz K. Mutations in the gene encoding lamin $A / C$ cause autosomal dominant Emery-Dreifuss muscular dystrophy. Nat Genet 1999;21: 285-8

Bonne G, Yaou RB, Beroud C, Boriani G, Brown S, de Visser $M$, Duboc D, Ellis J, Hausmanowa-Petrusewicz I, Lattanzi G, Merlini L, Morris G, Muntoni F, Opolski G, Pinto YM, Sangiuolo F, Toniolo D, Trembath R, van Berlo JH, van der Kooi AJ, Wehnert M. 108th ENMC International Workshop, 3rd Workshop of the MYO-CLUSTER project: EUROMEN, 7th International Emery-Dreifuss Muscular Dystrophy (EDMD) Workshop, 13-15 September 2002, Naarden, The Netherlands. Neuromuscul Disord 2003;13:508-15

Brodsky GL, Muntoni F, Miocic S, Sinagra G, Sewry C, Mestroni L. Lamin A/C gene mutation associated with dilated cardiomyopathy with variable skeletal muscle involvement. Circulation 2000;101:473-6

Burke B, Stewart CL. Life at the edge: the nuclear envelope and human disease. Nat Rev Mol Cell Biol 2002;3:575-85

Burkett EL, Hershberger RE. Clinical and genetic issues in familial dilated cardiomyoparhy. J Am Coll Cardiol 2005;45: 969-81

Fatkin D, MacRae C, Sasaki T, Wolff MR, Porcu M, Frenneaux M, Atherton J, Vidaillet HJ Jr, Spudich S, De Girolami U, Seidman JG, Seidman C, Muntoni F, Muehle G, Johnson W, McDonough $B$. Missense mutations in the rod domain of the lamin $A / C$ gene as causes of dilated cardiomyopathy and conduction-system disease. N Engl J Med 1999;341:1715-24

Grunig E, Tasman JA, Kucherer H, Franz W, Kubler W, Katus $\mathrm{HA}$. Frequency and phenotypes of familial dilated cardiomyopathy. J Am Coll Cardiol 1998;31:186-94

Hermida-Prieto M, Monserrat L, Castro-Beiras A, Laredo R, Soler R, Peteiro J, Rodriguez E, Bouzas B, Alvarez N, Muniz $\mathrm{J}$, Crespo-Leiro M. Familial dilated cardiomyopathy and isolated left ventricular noncompaction associated with lamin A/C gene mutations. Am J Cardiol 2004;94:50-4

Hershberger RE, Hanson E, Jakobs PM, Keegan H, Coates $\mathrm{K}$, Bousman S, Litt M. A novel lamin A/C mutation in a family with dilated cardiomyopathy, prominent conduction system disease, and need for permanent pacemaker implantation. Am Heart J 2002;144:1081-6

Jakobs PM, Hanson EL, Crispell KA, Toy W, Keegan H, Schilling K, Icenogle TB, Litt M, Hershberger RE. Novel lamin $\mathrm{A} / \mathrm{C}$ mutation in two families with dilated cardiomyopathy and conduction system disease. J Card Fail 2001;7:249-56

Karkkainen S, Helio T, Miettinen R, Tuomainen P, Peltola P, 
Rummukainen J, Ylitalo K, Kaartinen M, Kuusisto J, Toivonen L, Nieminen MS, Laakso M, Peuhkurinen K. A novel mutation, Ser143Pro, in the lamin A/C gene is common in finnish patients with familial dilated cardiomyopathy. Eur Heart J 2004; 25:885-93

Lee S, Hong S, Yoon Y, Yang I, Song K. Characterization of publicly available SNPs in the Korean population. Hum Mutat 2001;17:281-4

Mestroni L, Maisch B, McKenna WJ, Schwartz K, Charron P, Rocco C, Tesson F, Richter A, Wilke A, Komajda M. Guidelines for the study of familial dilated cardiomyopathies. Collaborative Research Group of the European Human and Capital Mobility Project on Familial Dilated Cardiomyopathy. Eur Heart J 1999;20:93-102

Mounkes LC, Kozlov SV, Rottman JN, Stewart CL. Expression of an LMNA-N195K variant of A-type lamins results in cardiac conduction defects and death in mice. Hum Mol Genet 2005; $14: 2167-80$
Raharjo WH, Enarson P, Sullivan T, Stewart CL, Burke B. Nuclear envelope defects associated with LMNA mutations cause dilated cardiomyopathy and Emery-Dreifuss muscular dystrophy. J Cell Sci 2001;114:4447-57

Sebillon P, Bouchier C, Bidot LD, Bonne G, Ahamed K, Charron $P$, Drouin-Garraud V, Millaire A, Desrumeaux G, Benaiche A, Charniot JC, Schwartz K, Villard E, Komajda M. Expanding the phenotype of LMNA mutations in dilated cardiomyopathy and functional consequences of these mutations. J Med Genet 2003;40:560-7

Taylor MR, Fain PR, Sinagra G, Robinson ML, Robertson AD, Carniel E, Di Lenarda A, Bohlmeyer TJ, Ferguson DA, Brodsky GL, Boucek MM, Lascor J, Moss AC, Li WL, Stetler GL, Muntoni F, Bristow MR, Mestroni L, Familial Dilated Cardiomyopathy Registry Research Group. Natural history of dilated cardiomyopathy due to lamin A/C gene mutations. J Am Coll Cardiol 2003;41:771-80 